\title{
Decomposition of the product of a monomial and a Demazure atom
}

\author{
Anna Ying Pun $\|^{t}$ \\ Department of Mathematics, University of Pennsylvania, Philadelphia, 19103, USA
}

\begin{abstract}
Abstract. We prove that the product of a monomial and a Demazure atom is a positive sum of Demazure atoms combinatorially. This result proves one particular case in a conjecture which provides an approach to a combinatorial proof of Schubert positivity property.

Résumé. Nous montrons combinatoirement que le produit d'un monôme et d'un atome de Demazure est une somme positive d'atomes de Demazure. Ce résultat montre un cas particulier d'une conjecture qui fournit une approche combinatoire de la propriété de positivité de Schubert.
\end{abstract}

Keywords. generalized Demazure atoms, key polynomials, Schubert positivity, nonsymmetric Macdonald polynomials, skyline filings

\section{Introduction}

Let $\mathbb{N}$ (or $\mathbb{Z}^{+}$) be the set of all positive integers and $\mathbb{Z}_{\geq 0}$ be the set of non-negative integers. Also we denote by $S_{k}$ the set of all permutations of the set $\{1, \ldots, k\}$. For $n \in \mathbb{Z}_{\geq 0}$ and $k \in \mathbb{N}$, we say $\alpha=$ $\left(\alpha_{1}, \alpha_{2}, \ldots, \alpha_{k}\right) \in\left(\mathbb{Z}_{\geq 0}\right)^{k}$ is a weak composition $n$ (denoted as $\alpha \vDash n$ ) with $k$ parts if $\sum_{i=1}^{k} \alpha_{i}=n$ and write $l(\alpha)=k$ to denote the length (the number of parts) of $\alpha$. Furthermore, if $\alpha_{1} \geq \alpha_{2} \geq \cdots \geq \alpha_{k} \geq 0$, we call $\alpha$ a partition of $n$ with $k$ parts and write $\alpha \vdash n$ (usually we denote $l(\alpha)=\max \left\{i \mid \alpha_{i}>0\right\}$ for $\alpha$ being a partition).

We denote $\bar{\alpha}$ as the reverse of $\alpha$, that is, $\bar{\alpha}=\left(\alpha_{k}, \ldots, \alpha_{1}\right)$. Define $\omega_{\alpha}$ as the permutation of minimal length such that $\omega_{\alpha}(\alpha):=\left(\alpha_{\omega_{\alpha}(1)}, \alpha_{\omega_{\alpha}(2)}, \ldots, \alpha_{\omega_{\alpha}(k)}\right)$ is a partition. Given two weak compositions $\alpha$ and $\beta$, we write $\beta \geq \alpha$ if and only if $\omega_{\beta} \leq \omega_{\alpha}$ in the strong Bruhat order.

Let $\alpha$ be a weak composition. The augmented diagram of shape $\alpha$ is the figure with $|\alpha|+l(\alpha)$ cells (or boxes) where column $i$ has $\alpha_{i}+1$ cells. The bottom row is called the basement of the augmented diagram.

For example, if $\alpha=(1,0,1,0,0,4,0,6,5)$, then the augmented diagram of $\alpha$ is

\footnotetext{
$\dagger^{\dagger}$ mail: punying@sas. upenn.edu

1365-8050 (c) 2016 Discrete Mathematics and Theoretical Computer Science (DMTCS), Nancy, France
} 


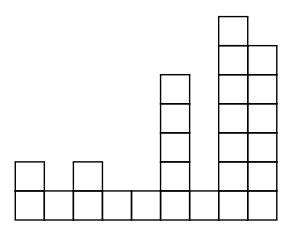

Also we impose an order, called the reading order, on the cells of the diagram which reads each row from left to right, starting from the top row and ends in the bottom row. So the order of the above diagram is:

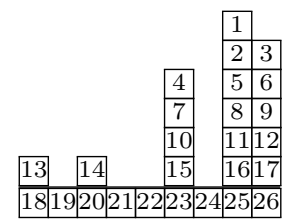

where the number in each cell represents the order of that cell in reading order.

A filling of an augmented diagram is an assignment of a positive integer to each cell in the diagram.

Macdonald [Mac96] defined a family of non-symmetric polynomials, called non-symmetric Macdonald polynomials,

$$
\left\{E_{\gamma}\left(x_{1}, \ldots, x_{n} ; q, t\right) \mid \gamma \text { is a weak composition with } n \text { parts, } n \in \mathbb{N}\right\}
$$

which shares many properties with the family of symmetric Macdonald polynomials [Mac95]

$$
\left\{P_{\lambda}\left(x_{1}, \ldots, x_{n} ; q, t\right) \mid \lambda \text { is a partition with } n \text { parts, } n \in \mathbb{N}\right\} .
$$

Haglund, Haiman and Loehr [HHL08] obtained a combinatorial formula for $E_{\gamma}\left(x_{1}, \ldots, x_{n} ; q, t\right)$ using fillings of augmented diagram of shape $\gamma$, called skyline fillings, satisfying certain constraints.

Marshall[Mar99] studied the family of non-symmetric Macdonald polynomials using another notation $\hat{E}_{\gamma}\left(x_{1}, \ldots, x_{n} ; q, t\right):=E_{\bar{\gamma}}\left(x_{n}, \ldots, x_{1} ; \frac{1}{q}, \frac{1}{t}\right)$. In particular, by setting $q=t=0$ in $\hat{E}_{\gamma}$, one can obtain Demazure atoms (first studied by Lascoux and Schützenberger[LS90]) $\mathcal{A}_{\gamma}=\hat{E}_{\gamma}\left(x_{1}, \ldots, x_{n} ; 0,0\right)=$ $E_{\bar{\gamma}}\left(x_{n}, \ldots, x_{1} ; \infty, \infty\right)$. Similarly, one can obtain Demazure characters (key polynomials) by setting $q=t=0$ in $E_{\gamma}$, i.e., $\kappa_{\gamma}=E_{\gamma}\left(x_{1}, \ldots, x_{n} ; 0,0\right)=\hat{E}_{\bar{\gamma}}\left(x_{n}, \ldots, x_{1} ; \infty, \infty\right)$. The set of all Demazure atoms forms a basis for the polynomial ring, as does the set of all key polynomials.

Haglund, Luoto, Mason, Remmel and van Willigenburg [HLMvW11], [HMR13] further studied the combinatorial formulas for Demazure atoms and Demazure characters given by the skyline fillings and obtained results which generalized those for Schurs functions like the Pieri Rule, the Robinson-SchenstedKnuth (RSK) algorithm, and the Littlewood-Richardson (LR) rule.

From now on we only consider fillings whose entries in each column are weakly decreasing from bottom (to top) since those are the fillings related to Demazure atoms and Demazure characters [HHL08].

For any two columns (including the basement cells) $i$ and $j$ with $i<j$, we pick three cells $X, Y$ and $Z$, where cell $X$ is immediately above cell $Y$ in the 'taller' column $k$, where

$$
k=\left\{\begin{array}{lll}
i & \text { if } \quad \alpha_{i} \geq \alpha_{j} \\
j & \text { if } \quad \alpha_{i}<\alpha_{j}
\end{array}\right.
$$


and cell $Z$ from the 'shorter' column to form a triple $(X, Y, Z)$ in the following way:

$$
\left\{\begin{array}{ll}
\text { Type A triple: cell } Z \text { is in the same row as cell } X & \text { if } \quad \alpha_{i} \geq \alpha_{j} \\
\text { Type B triple: cell } Z \text { is in the same row as cell } Y & \text { if } \quad \alpha_{i}<\alpha_{j}
\end{array} .\right.
$$

We say $(X, Y, Z)$ forms a coinversion triple if the filling $F$ of the diagram assigns each cell in the triple a positive integer, say $F(X), F(Y), F(Z)$ respectively, in such a way that $F(X) \leq F(Z) \leq F(Y)$. Otherwise we call $(X, Y, Z)$ an inversion triple.

Here are some examples of triples:
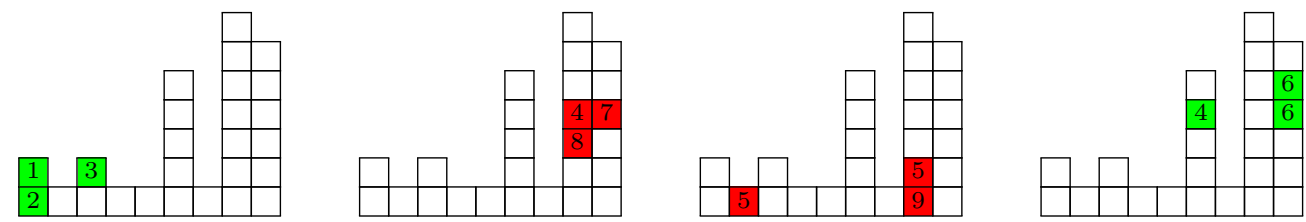

The first two are type A triples and the last two are type B triples while the second and the third ones are coinversion triples and the first and the last one are inversion triples.

A semi-standard augmented filling (SSAF) of an augmented diagram with shape being a weak composition $\alpha=\left(\alpha_{1}, \alpha_{2}, \ldots, \alpha_{k}\right)$ is a filling satisfying:

1. the basement entries form a permutation $\sigma$ (in one line notation) of $\{1, \ldots, k\}$, i.e., $\sigma \in S_{k}$;

2. every (Type A or B) triple is an inversion triple.

We denote $\operatorname{SSAF}(\sigma, \alpha)$ the set of all SSAF of an augmented diagram of shape $\alpha=\left(\alpha_{1}, \alpha_{2}, \ldots, \alpha_{k}\right)$ with basement entries (from left to right) being $\sigma \in S_{k}$ (i.e., basement of column $i$ has entry $\sigma(i)$ ).

The following combinatorial formulas can be found in [HHL08, LS90, Mas09].

1. Let $\mathcal{A}_{\alpha}$ be the Demazure atom of shape $\alpha$. Then

$$
\mathcal{A}_{\alpha}=\sum_{F \in S S A F\left(\epsilon_{k}, \alpha\right)} x^{F}
$$

where $\epsilon_{k} \in S_{k}$ is the identity. In particular, if $\alpha$ is a partition, then $\mathcal{A}_{\alpha}=x^{\alpha}=\prod_{i=1}^{k} x_{i}^{\alpha_{i}}$ is a monomial.

2. Let $\kappa_{\bar{\alpha}}$ be the key polynomial (Demazure character) of shape $\alpha$. Then

$$
\kappa_{\bar{\alpha}}=\sum_{F \in S S A F\left(\bar{\epsilon}_{k}, \alpha\right)} x^{F}
$$

where $\bar{\epsilon}_{k}(i)=k+1-i$. 
Note that if $\bar{\alpha}$ is a partition, $\kappa_{\alpha}=\mathcal{A}_{\bar{\alpha}}$ which is a monomial by 1

The following are some properties among keys and atoms and their products:

3. A key polynomial is a positive sum of Demazure atoms. In fact,

$$
\kappa_{\bar{\alpha}}=\sum_{\beta \geq \alpha} \mathcal{A}_{\beta} .
$$

4. A key polynomial with a partition shape $\lambda$, with $l(\lambda)=k$, is the Schur polynomial $s_{\lambda}$, i.e., $\kappa_{\bar{\lambda}}=$ $s_{\lambda}\left(x_{1}, \ldots, x_{k}\right)$.

Let $\lambda, \mu$ be partitions and $\alpha, \beta$ be weak compositions. Denote $+_{\mathcal{A}}$ and $+_{\kappa}$ as being able to be decomposed into positive sum of atoms and keys respectively. Note that by 3 , $+_{\kappa}$ implies $+_{\mathcal{A}}$. Otherwise, we put a $\times$ in the cell. For example, a partition $(\mu)$-shaped atom times a key of any shape $(\alpha)$ is key positive and hence we put $+_{\kappa}$ in the corresponding box.

\begin{tabular}{|c|c|c|c|c|c|}
\cline { 2 - 6 } \multicolumn{1}{c|}{} & \multicolumn{2}{c|}{ Atoms } & \multicolumn{2}{c|}{ Keys } \\
\cline { 2 - 6 } \multicolumn{1}{c|}{} & shape & $\lambda$ & $\alpha$ & $\lambda$ & $\alpha$ \\
\hline \multirow{2}{*}{ Atoms } & $\mu$ & $+_{\mathcal{A}}$ & $+_{\mathcal{A}}(1)$ & $+_{\mathcal{A}}$ & $+_{\kappa}(2)$ \\
\cline { 2 - 6 } & $\beta$ & $\times$ & $+_{\mathcal{A}}$ & $\times$ \\
\hline \multirow{2}{*}{ Keys } & $\mu$ & & & $+_{\kappa}$ & $+_{\kappa}$ \\
\cline { 2 - 6 } & $\beta$ & & & open 3 \\
\hline
\end{tabular}

Tab. 1: Decomposition of products of atoms and keys into atoms

The positive results in the table can be found in [HLMvW11], except for the cells marked (1), (2) and (3).

This paper proves (1) combinatorially in Section 4 which was open:

Theorem 1 The product $\mathcal{A}_{\mu} \cdot \mathcal{A}_{\alpha}$ is atom-positive for any partition $\mu$ and weak composition $\alpha$.

The coefficients in the decomposition into atoms are actually counting the number of ways to insert words arising from SSAF of shape $\alpha$ into a SSAF of shape $\mu$ and we will discuss properties of words and how to record different ways of insertion in Section 2 and 3 . Also note that the product in the theorem is not key positive. A simple counter example would be just putting $\mu$ as the empty partition, that is, with all entries 0 .

(2) is proved in [Jos03] (the proof involves crystals but does not involve SSAF). Both results (1) and (2) imply $+_{\mathcal{A}}$ for the $\mathcal{A}_{\mu} \cdot \kappa_{\bar{\alpha}}$ cell. Also we conjecture that we can apply the bijection in the proof in Theorem 6.1 in [HLMvW11] on Theorem 13 in Section 4 to give a tableau-combinatorial proof to (2).

As for the product of two keys of arbitrary shapes, that is, the cell marked (3), there are examples showing that the product of two key polynomials of arbitrary shapes is not a positive sum of keys. So it remains to check if it is a positive sum of atoms, which is still open. Hence (3) gives the following conjecture which we have verified for $l(\alpha), l(\beta) \leq 3$ : 
Conjecture 1 Let $\alpha, \beta$ be weak compositions. Then the product of the key polynomials of shape $\bar{\alpha}$ and $\bar{\beta}$ can be written as a positive sum of atoms, i.e.,

$$
\kappa_{\alpha} \cdot \kappa_{\beta}=\sum_{\gamma \vDash|\alpha|+|\beta|} c_{\alpha \beta}^{\gamma} \mathcal{A}_{\gamma}
$$

for some non-negative integers $c_{\alpha \beta}^{\gamma}$.

It is a classical result in Algebraic Geometry that the product of two Schubert polynomials can be written as a positive sum of Schubert polynomials. A representation theoretic proof is also given recently by using Kráskiewicz-Pragacz modules [Wat14]. However a combinatorial proof of the positivity property of Schubert polynomials has long been open. Since every Schubert polynomial is a positive sum of key polynomials [LS89], the product of two Schubert polynomials is a positive sum of product of two key polynomials and hence by Conjecture 1 is a positive sum of atoms. This provides a possible approach to a combinatorial proof of the positivity property of Schubert polynomials by trying to recombine the atoms into keys and hence into Schubert polynomials.

Since words and insertion are the crucial tools used to prove Theorem 13 , we will discuss an algorithm on words in Section 2, then we will relate it to SSAF and recording tableaux in Section 3 . Finally we will use the results we obtained in Section 2 and 3 to prove the main theorem that the product of $\mathcal{A}_{\lambda} \cdot \mathcal{A}_{\alpha}$ is atom-positive for partition $\lambda$ and weak composition $\alpha$ in Section 4

\section{Convert a column word to a row word}

Definition 1 A word is a sequence of positive integers.

Definition 2 Let $a, b, c \in \mathbb{N}$ and $u, v$ be some fixed (can be empty) words. Define twisted Knuth relation $\sim^{*}$ by:

$$
\begin{array}{ll}
\text { 1. ubacv } \sim^{*} u b c a v & \text { if } c \leq b<a \\
\text { 2. uacbv } \sim^{*} u c a b v & \text { if } c<b \leq a .
\end{array}
$$

Then we say two words $w$ and $w^{\prime}$ are twisted Knuth equivalent if $w$ can be transformed to $w^{\prime}$ by repeated use of 1. and 2. and we write $w \sim^{*} w^{\prime}$.

Definition 3 A word $w$ is a column word if it can be broken down into $k$ weakly decreasing subsequences of weakly decreasing lengths

$$
w=a_{11} \ldots a_{1 c_{1}}\left|a_{21} \ldots a_{2 c_{2}}\right| \cdots \mid a_{k 1} \ldots a_{k c_{k}}
$$

where $c_{1} \geq c_{2} \geq \cdots \geq c_{k}>0, c_{1}, \ldots, c_{k} \in \mathbb{N}$ such that

$$
\begin{cases}a_{i j} \geq a_{i, j+1} & \text { for } 1 \leq j<c_{i}, 1 \leq i \leq k \\ a_{i+1, c_{i+1}-j}>a_{i, c_{i}-j} & \text { for } 0 \leq j<c_{i}, 1 \leq i<k\end{cases}
$$

Definition $4 A$ word $w$ is a row word if it can be broken down into $k$ strictly increasing subsequences of weakly decreasing lengths

$$
w=a_{11} \ldots a_{1 r_{1}}\left|a_{21} \ldots a_{2 r_{2}}\right| \ldots \mid a_{k 1} \ldots a_{k r_{k}}
$$


where $r_{1} \geq r_{2} \geq \cdots \geq r_{k}>0, r_{1}, \ldots, r_{k} \in \mathbb{N}$ such that

$\begin{cases}a_{i j}<a_{i, j+1} & \text { for } 1 \leq j<r_{i}, 1 \leq i \leq k \\ a_{i+1, r_{i+1}-j} \leq a_{i, r_{i}-j} & \text { for } 0 \leq j<r_{i}, 1 \leq i<k\end{cases}$

Given a SSAF, one can get its column word by using the algorithm described in [HLMvW11], while one can get its row word by reading the entries of each row in ascending order, starting from the bottom row to the top row. We call a word a column (row resp.) word because when we insert each subsequence of the word using the insertion in [Mas08], a new column (row resp.) will be created.

Example $1886531|97643| 9764|5| 6$ is a column word whose corresponding SSAF is:

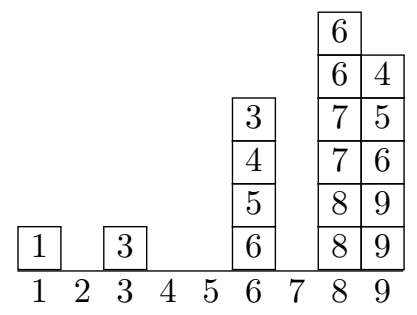

and the corresponding row word is $13689|589| 467|357| 46 \mid 6$.

Lemma 2 Let $u:=a_{1} a_{2} \ldots a_{n} \mid b$ and $v:=a_{1} a_{2} \ldots a_{n}|b| c$ be two column words, where $c>b>a_{n}$, $n \in \mathbb{N}$. Then

1. $u \sim^{*} b^{\prime} a_{1}^{\prime} \ldots a_{n}^{\prime}$ where $b^{\prime}<a_{1}^{\prime}, a_{1}^{\prime} \geq a_{2}^{\prime} \geq \cdots \geq a_{n}^{\prime}$, and $b^{\prime}:=a_{t}$ where $t=\min \left\{j \mid b>a_{j}\right\}$,

2. $v \sim^{*} b^{\prime} c^{\prime} a_{1}^{\prime \prime} a_{2}^{\prime \prime} \ldots a_{n}^{\prime \prime}$ where $b^{\prime}$ is defined as in $1 ., b^{\prime}<c^{\prime}<a_{1}^{\prime \prime}$ and $a_{1}^{\prime \prime} \geq a_{2}^{\prime \prime} \geq \cdots \geq a_{n}^{\prime \prime}$.

Lemma 3 Let $k \geq 2$ be an integer and $i_{1}, \ldots, i_{k}$ be nonnegative integers. Let

$$
w_{0}=a_{11}^{\left(i_{1}\right)} \ldots a_{1 c_{1}}^{\left(i_{1}\right)}\left|a_{21}^{\left(i_{2}\right)} \ldots a_{2 c_{2}}^{\left(i_{2}\right)}\right| \ldots \mid a_{k 1}^{\left(i_{k}\right)} \ldots a_{k c_{k}}^{\left(i_{k}\right)}
$$

be a column word. Then there exist $a_{r s}^{\left(i_{r}+1\right)}$ where $1 \leq s \leq c_{r}, 1 \leq r<k$ and $a_{k 1}^{\left(i_{k}+m\right)}$ where $1 \leq m<k$ satisfying

$$
\left\{\begin{array}{l}
a_{k-j-1, c_{k-j-1}}^{\left(i_{k-j-1}\right)}<a_{k 1}^{\left(i_{k}+j\right)}<a_{k-j, 1}^{\left(i_{k-j}+1\right)} \quad \text { for } 1 \leq j<k-1 \quad(k>3) \\
a_{k 1}^{\left(i_{k}+k-1\right)}<a_{11}^{\left(i_{1}+1\right)} \\
a_{1,1}^{\left(i_{1}+1\right)} \ldots a_{1, c_{1}}^{\left(i_{1}+1\right)}|\ldots| a_{k-1,1}^{\left(i_{k-1}+1\right)} \ldots a_{k-1, c_{k-1}}^{\left(i_{k-1}+1\right)} \mid a_{k 2}^{\left(i_{k}\right)} \ldots a_{k c_{k}}^{\left(i_{k}\right)} \quad \text { is a column word }
\end{array}\right.
$$

such that $w_{0} \backsim^{*} w_{1} \sim^{*} \ldots \sim^{*} w_{k-1}$, where

$$
\left\{\begin{array}{c}
w_{j}:=a_{11}^{\left(i_{1}\right)} \ldots a_{1 c_{1}}^{\left(i_{1}\right)} \ldots a_{k-j-1,1}^{\left(i_{k-j-1}\right)} \ldots a_{k-j-1, c_{k-j-1}}^{\left(i_{k-j}\right)} a_{k 1}^{\left(i_{k}+j\right)} a_{k-j, 1}^{\left(i_{k-j}+1\right)} \ldots a_{k-j, c_{k-j}}^{\left(i_{k-j}+1\right)} \ldots \\
\ldots a_{k-1,1}^{\left(i_{k-1}+1\right)} \ldots a_{k-1, c_{k-1}}^{\left(i_{k-1}+1\right)} a_{k 2}^{\left(i_{k}\right)} \ldots a_{k c_{k}}^{\left(i_{k}\right)} \quad \text { for } 1 \leq j<k-1 \\
w_{k-1}:=a_{k 1}^{i_{k}+k-1} a_{11}^{\left(i_{1}+1\right)} \ldots a_{1 c_{1}}^{\left(i_{1}+1\right)} \ldots a_{k-1,1}^{\left(i_{k-1}+1\right)} \ldots a_{k-1, c_{k-1}}^{\left(i_{k-1}+1\right)} a_{k 2}^{\left(i_{k}\right)} \ldots a_{k c_{k}}^{\left(i_{k}\right)} .
\end{array}\right.
$$


The proof is basically induction on $k$.

Lemma 3 shows how to create the first entry of the corresponding row word, leaving the remaining part as a column word. We illustrate the Lemma by an example with $a_{k 1}^{\left(i_{k}+j\right)}$ circled for all $0 \leq j \leq k-1$.

Example 2 Let $w_{0}$ be the word in Example 1 , hence $k=5, c_{1}=6, c_{2}=5, c_{3}=4, c_{4}=c_{5}=1$. Set $i_{j}=0$ for all $j$. Then we have

$$
\left\{\begin{array}{l}
w_{0}=a_{11}^{(0)} \ldots a_{16}^{(0)}\left|a_{21}^{(0)} \ldots a_{25}^{(0)}\right| a_{31}^{(0)} \ldots a_{34}^{(0)}\left|a_{41}^{(0)}\right| a_{51}^{(0)}=886531|97643| 9764|5| 6 \\
w_{1}=a_{11}^{(0)} \ldots a_{16}^{(0)}\left|a_{21}^{(0)} \ldots a_{25}^{(0)}\right| a_{31}^{(0)} \ldots a_{34}^{(0)}\left|a_{51}^{(1)}\right| a_{41}^{(1)}=886531|97643| 9764 \mid(5 \mid 6 \\
w_{2}=a_{11}^{(0)} \ldots a_{16}^{(0)}\left|a_{21}^{(0)} \ldots a_{25}^{(0)}\right| a_{51}^{(2)}\left|a_{31}^{(1)} \ldots a_{34}^{(1)}\right| a_{41}^{(1)}=886531|97643|(4|9765| 6 \\
w_{3}=a_{11}^{(0)} \ldots a_{16}^{(0)}\left|a_{51}^{(3)}\right| a_{21}^{(1)} \ldots a_{25}^{(1)}\left|a_{31}^{(1)} \ldots a_{34}^{(1)}\right| a_{41}^{(1)}=886531|3| 97644|9765| 6 \\
w_{4}=a_{51}^{(4)}\left|a_{11}^{(1)} \ldots a_{16}^{(1)}\right| a_{21}^{(1)} \ldots a_{25}^{(1)}\left|a_{31}^{(1)} \ldots a_{34}^{(1)}\right| a_{41}^{(1)}=1|886533| 97644|9765| 6
\end{array}\right.
$$

Lemma 4 Let $w=a_{11} \ldots a_{1 c_{1}} \ldots a_{k 1} \ldots a_{k c_{k}}$ be a column word. Then there exists a sequence $\left\{b_{i j}\right\}_{1 \leq j \leq c_{i}, 1 \leq i \leq k}$ such that $w \sim^{*} b_{k 1} b_{k-1,1} \ldots b_{11} b_{12} \ldots b_{1 c_{1}} b_{22} \ldots b_{2 c_{2}} \ldots b_{k 2} \ldots b_{k c_{k}}$, where $b_{11}>b_{21}>\cdots>b_{k 1}$ and $b_{11} \ldots b_{1 c_{1}}\left|b_{22} \ldots b_{2 c_{2}}\right| \cdots \mid b_{k 2} \ldots b_{k c_{k}}$ is a column word (with lengths $c_{1}>c_{2}-1 \geq \cdots \geq c_{k}-1$ ).

The proof is applying Lemma 3 inductively.

Using the notation in Lemma 4, since $w \sim^{*} b_{k 1} b_{k-1,1} \ldots b_{11} b_{12} \ldots b_{1 c_{1}} b_{22} \ldots b_{2 c_{2}} \ldots b_{k 2} \ldots b_{k c_{k}}$, where $b_{11}>b_{21}>\cdots>b_{k 1}$, the SSAF with basement being the identity (i.e., $12 \cdots n$, for some positive integer $n$ ) representing the word $w$ is the same as that representing $b_{k 1} b_{k-1,1} \ldots b_{11} b_{12} \ldots b_{1 c_{1}} b_{22} \ldots b_{2 c_{2}} \ldots b_{k 2} \ldots b_{k c_{k}}$. Denote $F(w)$ as the SSAF created.

Since $b_{k 1} b_{k-1,1} \ldots b_{11}$ is strictly increasing, by Lemma 15 in [HMR13], they create new cells in ascending reading order (i.e., one after another) and hence is exactly the first entire row of $F(w)$ as the entries are fixed when inserting $b_{k 1} b_{k-1,1} \ldots b_{11}$ into an empty atom, and there are $k$ columns (as $w$ has $k$ subsequences) and so the first row has length $k$ and hence the row reading word has exactly $b_{k 1}, b_{k-1,1}, \ldots, b_{11}$ as the first subsequence. That means we can apply Lemma 4 to find the first subsequence of the row reading word of $F(w)$.

Since $b_{12} \ldots b_{1 c_{1}} b_{22} \ldots b_{2 c_{2}} \ldots b_{k 2} \ldots b_{k c_{k}}$ is a column word, we can apply Lemma 4 again and get the second subsequence of the row reading word of $F(w)$, and we can apply Lemma 4 repeatedly on the remaining $b_{i j}$ 's until we get all the subsequences of the row reading word of $F(w)$. As a result, we can convert $w$ into the row reading word of $F(w)$ by applying Lemma 4 repeatedly as described.

\section{Convert a Column Recording Tableau to a Row Recording Tableau}

This section gives an interpretation of the twisted Knuth equivalence using recording tableaux. We use the insertion in [Mas08] and the generalized Littlewood-Richardson rule in [HLMvW11]. We also use 
the notation $U \leftarrow W$ for a SSAF $U$ and a biword $W=\left(\begin{array}{lll}x_{1} & x_{2} & \ldots x_{n} \\ y_{1} & y_{2} & \ldots y_{n}\end{array}\right)$ for some integer $n$ to denote the pair $\left(U^{\prime}, L\right)$ where $U^{\prime}$ is the SSAF obtained by $U \leftarrow y_{1} y_{2} \ldots y_{n}$ while $L$ is the recording tableau, i.e., by putting $x_{i}$ into the cell created when $y_{i}$ is being inserted. In particular, if $y_{1} y_{2} \ldots y_{n}$ is a column (row resp.) word, then we call $L$ as a column (row resp.) recording tableau. By abuse of notation, we sometimes refer $U \leftarrow W$ to either $U^{\prime}$ or $L$ (depending on the context). Note that if we change the basement $\epsilon_{n}$ into the large basement in [HLMvW11], a column recording tableau is the same as an LRS in [HLMvW11].

Lemma 5 Let $U$ be a SSAF with basement $\epsilon_{n}$ and shape $\alpha$ for some positive integer $n$ and $l(\alpha) \leq n$. Consider the biword $W=\left(\begin{array}{lll}2 & 2 & 1 \\ a & b & c\end{array}\right), a \geq b, c>b$ (i.e., ab $\mid c$ is a column word.). Let L be the recording tableau of $U \leftarrow W$. Let $V$ be the SSAF representing the word abc and $a^{\prime} b^{\prime} c^{\prime}$ be the row reading word of $V\left(\right.$ so $a^{\prime}<b^{\prime}$ and $c^{\prime} \leq b^{\prime}$ and $\left.a b c \sim^{*} a^{\prime} b^{\prime} c^{\prime}\right)$. Consider the biword $\widetilde{W}=\left(\begin{array}{ccc}1 & 1 & 2 \\ a^{\prime} & b^{\prime} & c^{\prime}\end{array}\right)$ and let $\widetilde{L}$ be the recording tableau of $U \leftarrow \widetilde{W}$. Then $L$ determines $\widetilde{L}$.

Lemma 6 Let $U$ be a SSAF with basement $\epsilon_{n}$ and shape $\alpha$ for some positive integer $n$ and $l(\alpha) \leq n$. Consider the biword $W=\left(\begin{array}{ccccc}2 & 2 & \cdots & 2 & 1 \\ a_{1} & a_{2} & \cdots & a_{k} & b\end{array}\right), a_{1} \geq a_{2} \geq \cdots \geq a_{k}, b>a_{k}\left(\right.$ i.e., $a_{1} a_{2} \ldots a_{k} \mid b$ is $a$ column word ). Let $L$ be the recording tableau of $U \leftarrow W$. If $b>a_{1}$, then $L$ has reading word $22 \ldots 21$, i.e., $b$ creates the last cell in reading order and the insertion of $a_{2} \ldots a_{k}$ is independent of the insertion of b.

Lemma 6 follows by applying Lemma 5 inductively.

Lemma 7 Let $U$ be a SSAF with basement $\epsilon_{n}$ and shape $\alpha$ for some positive integer $n$ and $l(\alpha) \leq n$. Consider the biword $W=\left(\begin{array}{ccccc}2 & 2 & \cdots & 2 & 1 \\ a_{1} & a_{2} & \cdots & a_{k} & b\end{array}\right), a_{1} \geq a_{2} \geq \cdots \geq a_{k}, b>a_{k}\left(\right.$ i.e., $a_{1} a_{2} \ldots a_{k} \mid b$ is a column word ). Let $L$ be the recording tableau of $U \leftarrow W$. If $i=\min \left\{j \mid b>a_{j}\right\}$, then the cell created by inserting $a_{m}$ for $m>i$ is not affected by the insertion of $b$.

Proof: Since $U \leftarrow a_{1} a_{2} \ldots a_{k} b=\left(U \leftarrow a_{1} a_{2} \ldots a_{i-1}\right) \leftarrow a_{i} \ldots a_{k} b$. By applying Lemma 6 with the $U$ in the lemma being $U \leftarrow a_{1} \ldots a_{i-1}$ and result follows.

Lemma 8 Let $U$ be a SSAF with basement $\epsilon_{n}$ and shape $\alpha$ for some positive integer $n$ and $l(\alpha) \leq n$. Consider the biword $W=\left(\begin{array}{ccccc}2 & 2 & \cdots & 2 & 1 \\ a_{1} & a_{2} & \cdots & a_{k} & b\end{array}\right), a_{1} \geq a_{2} \geq \cdots \geq a_{k}, b>a_{k}\left(\right.$ i.e., $a_{1} a_{2} \ldots a_{k} \mid b$ is $a$ column word ). Let $L$ be the recording tableau of $U \leftarrow W$. Suppose $b \leq a_{k-1}$. Let $\widetilde{L}$ be the recording tableau of $U \leftarrow \widetilde{W}$ where $\widetilde{W}=\left(\begin{array}{cccccc}1 & 1 & 2 & \cdots & 2 & 2 \\ a_{k} & a_{1} & a_{2} & \cdots & a_{k-1} & b\end{array}\right)$, then $L$ determines $\widetilde{L}$.

The above result follows by applying Lemma 5 inductively.

Lemma 9 Let $U$ be a SSAF with basement $\epsilon_{n}$ and shape $\alpha$ for some positive integer $n$ and $l(\alpha) \leq n$. Consider the biword $W=\left(\begin{array}{ccccc}2 & 2 & \cdots & 2 & 1 \\ a_{1} & a_{2} & \cdots & a_{k} & b\end{array}\right), a_{1} \geq a_{2} \geq \cdots \geq a_{k}, b>a_{k}\left(i . e ., a_{1} a_{2} \ldots a_{k} \mid b\right.$ 
is a column word ). Let $L$ be the recording tableau of $U \leftarrow W$. Let $\widetilde{L}$ be the recording tableau of $U \leftarrow \widetilde{W}$ where $\widetilde{W}=\left(\begin{array}{ccccc}1 & 1 & 2 & \cdots & 2 \\ b^{\prime} & a_{1}^{\prime} & a_{2}^{\prime} & \cdots & a_{k}^{\prime}\end{array}\right)$, such that $b^{\prime}=a_{i}$, where $i=\min \left\{j \mid b>a_{j}\right\}$ and $b^{\prime}<a_{1}^{\prime}, a_{1}^{\prime} \geq a_{2}^{\prime} \geq \cdots \geq a_{k}^{\prime}$ (one can verify that $b^{\prime} a_{1}^{\prime} \ldots a_{k}^{\prime}$ is indeed the row reading word of the SSAF representing the word $a_{1} a_{2} \ldots a_{k}$ by 1 . in Lemma 2 , then $L$ determines $\widetilde{L}$.

Proof: If $i=k$, then we are done by Lemma 8 .

If $i=1$, then by 1 . in Lemma 2, we have $b^{\prime} a_{1}^{\prime} \ldots a_{k}^{\prime}=a_{1} b a_{2} \ldots a_{k}$ and hence by Lemma $6, b$ would create the last cell in reading order in $\widetilde{L}$ when being inserted and hence we know that which two cells are created by $a_{1}$ and $b$ in $\widetilde{L}$ and hence we know how to label the entries by marking those two cells as 1 and the rest as 2 .

Suppose $i<k$ then by 1 . in Lemma 2, we have $b^{\prime} a_{1}^{\prime} \ldots a_{k}^{\prime}=a_{i} a_{1} \ldots a_{i-1} b a_{i+1} \ldots a_{k}$. Now by Lemma 7. we know all $a_{j}$, where $j>i$, create the same cells in $L$ and $\widetilde{L}$ and since $a_{1} \ldots a_{i-1} a_{i} b \sim^{*}$ $a_{i} a_{1} \ldots a_{i-1} b$, we just need to first apply Lemma 8 on $U \leftarrow a_{1} \ldots a_{i} b$ as the insertion recorded by $L$ in the lemma in order to find the first $(i+1)$ cells created in $\widetilde{L}$ by the insertion $U \leftarrow a_{i} a_{1} \ldots a_{i-1} b$ and then label the rest of cells created by $\left(U \leftarrow a_{i} a_{1} \ldots a_{i-1} b\right) \leftarrow a_{i+1} \ldots a_{k}$ as 2 and get the entries of $\widetilde{L}$ and result follows.

Lemma 9 gives a recording tableau interpretation of 1 . in Lemma 2 using $L$ and $\widetilde{L}$.

Lemma 10 Let $U$ be a SSAF with basement $\epsilon_{n}$ and shape $\alpha$ for some positive integer $n$ and $l(\alpha) \leq n$. Let $w_{0}=a_{11} \ldots a_{1 c_{1}}\left|a_{21} \ldots a_{2 c_{2}}\right| \ldots \mid a_{k 1} \ldots a_{k c_{k}}$ with $k \leq n$ be a column word and using the notation in Lemma 3 while we assume $i_{j}=0$ for $1 \leq j \leq c_{r}, 1 \leq r \leq k$, the recording tableau $L$ of $U \leftarrow W_{0}$ determines the recording tableau $\widetilde{L}_{m}$ of $U \leftarrow W_{m}$ for $0 \leq m \leq k-1$, where $L:=\widetilde{L}_{0}$ and $W_{m}$ is a biword with the lower word being $w_{m}$ and the upper word has entry $k+1-t$ if the lower word entry just below it is $a_{t j}^{(s)}$ for $s=i_{t}, i_{t}+1$, and $1_{(1)}$ if $m>0$ and the entry is $a_{k 1}^{\left(i_{k}+m\right)}$.

Lemma 10 follows by inductively using Lemma 9

Lemma 11 Let $U$ be a SSAF with basement $\epsilon_{n}$ and shape $\alpha$ for some positive integer $n$ and $l(\alpha) \leq n$. Let $w=a_{11} \ldots a_{1 c_{1}}\left|a_{21} \ldots a_{2 c_{2}}\right| \cdots \mid a_{k 1} \ldots a_{k c_{k}}$ with $k \leq n$ be a column word and using the notation in Lemma 4 the recording tableau $L$ of $U \leftarrow W$ determines the recording tableau $\widetilde{L}$ of $U \leftarrow \widetilde{W}$, where

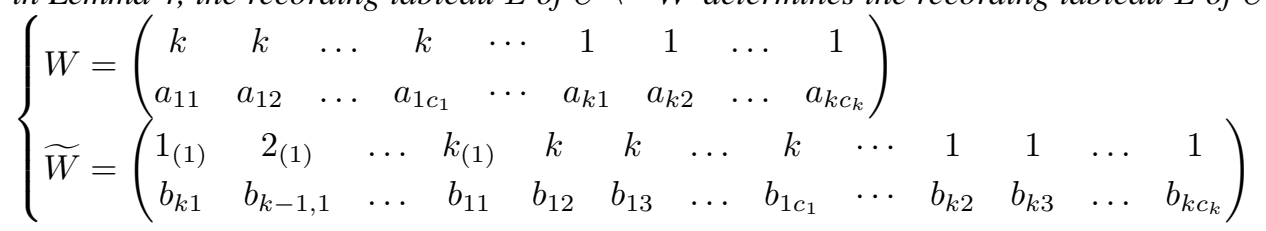

We apply Lemma 10 (which is like the tableaux version of Lemma 3 to get the result above.

Lemma 12 Let $U$ be a SSAF with basement $\epsilon_{n}$ and shape $\alpha$ for some positive integer $n$ and $l(\alpha) \leq n$. Let $w=a_{11} \ldots a_{1 c_{1}}\left|a_{21} \ldots a_{2 c_{2}}\right| \cdots \mid a_{k 1} \ldots a_{k c_{k}}$ with $k \leq n$ be a column word. Let $V$ be the SSAF representing $w$ (i.e., inserting $w$ into an empty atom). Let $\tilde{w}=x_{11} x_{12} \ldots x_{r 1} \cdots x_{c_{1}, 1} \ldots x_{c_{1}, r_{c_{1}}}$ (as $V$ has $c_{1}$ rows and $k$ columns and so $r_{1}$ is $k$ ), where $k=r_{1} \geq r_{2} \geq \cdots \geq r_{c_{1}}>0$ are the row lengths of $V$ from bottom to top, be the row reading word of $V$. Then the recording tableau $L$ of $U \leftarrow W$ determines the recording tableau $R$ of $U \leftarrow \widetilde{W}$, where 


$$
\left\{\begin{array}{l}
W=\left(\begin{array}{ccccccccc}
k & k & \ldots & k & \ldots & 1 & 1 & \ldots & 1 \\
a_{11} & a_{12} & \ldots & a_{1 c_{1}} & \ldots & a_{k 1} & a_{k 2} & \ldots & a_{k c_{k}}
\end{array}\right) \\
\widetilde{W}=\left(\begin{array}{ccccccccc}
1 & 1 & \ldots & 1 & \ldots & c_{1} & c_{1} & \ldots & c_{1} \\
x_{11} & x_{12} & \ldots & x_{1 r_{1}} & \ldots & x_{c_{1}, 1} & x_{c_{1}, 2} & \ldots & x_{c_{1}, r_{c_{1}}}
\end{array}\right)
\end{array}\right.
$$

Proof:

By Lemma 11 and the corresponding word in Lemma 4, given $L$, we know how to enter all the 1's in $R$, which are those cells marked $1_{(1)}, 2_{(1)}, \ldots, k_{(1)}$ after applying Lemma 11 as the $b_{k 1} b_{k-1,1} \ldots b_{11}=$ $x_{11} x_{12} \ldots x_{1 r_{1}}$ (by the argument after Lemma 4).

We remove the cells from $\widetilde{L}$ in Lemma 11 to create a new $L$ to apply Lemma 11 on, we can get the second row entries (as described in the paragraphs after Lemma 4), and hence we know how to put all the 2 's into $R$. By the same argument, we can fill in all entries in $R$ and hence $L$ determines $R$.

\section{Decomposition of the product of a monomial and an atom into positive sum of atoms}

We prove Theorem 1 mentioned in the Introduction. We rephrase the statement more precisely as follows.

Theorem 13 Let $\lambda$ be a partition and $\alpha$ be a weak composition. Let $\mathcal{A}_{\lambda}$ and $\mathcal{A}_{\alpha}$ be atoms of shape $\lambda$ and $\alpha$ respectively. Then

$$
\mathcal{A}_{\lambda} \cdot \mathcal{A}_{\alpha}=\sum_{\beta \models|\lambda|+|\alpha|, \lambda \subseteq \beta} c_{\lambda \alpha}^{\beta} \mathcal{A}_{\beta}
$$

where $c_{\lambda \alpha}^{\beta}$ is the number of distinct LRS of shape $\beta / \lambda$ created by column words whose corresponding SSAF has shape $\alpha$.

Proof: Since $\mathcal{A}_{\lambda}=x^{\lambda}$ (there is exactly one SSAF with shape $\lambda$, denoted by $U_{\lambda}$ ) and $\mathcal{A}_{\alpha}=\sum_{F \in \operatorname{SSAF}\left(\epsilon_{l(\alpha)}, \alpha\right)} x^{F}$, we have

$$
\mathcal{A}_{\lambda} \cdot \mathcal{A}_{\alpha}=\sum_{F \in S S A F\left(\epsilon_{l(\alpha)}, \alpha\right)} x^{\lambda} x^{F}
$$

To prove the theorem, we only need to check that given a LRS $L$ of shape $\beta / \lambda$ created by column words whose corresponding SSAF has shape $\alpha$, if there is some column word

$$
w=a_{11} \ldots a_{1 c_{1}}\left|a_{21} \ldots a_{2 c_{2}}\right| \cdots \mid a_{k 1} \ldots a_{k c_{k}}
$$

and biword

$$
W=\left(\begin{array}{ccccccccc}
k & k & \ldots & k & \ldots & 1 & 1 & \ldots & 1 \\
a_{11} & a_{12} & \ldots & a_{1 c_{1}} & \cdots & a_{k 1} & a_{k 2} & \ldots & a_{k c_{k}}
\end{array}\right)
$$


such that $U_{\lambda} \leftarrow W$ creates the same $L$, then the SSAF corresponding to $w$ also has shape $\alpha$.

First consider the last cell in reading order among all those containing entry $k$ in $L$, that means it is the very first entry inserted. Since $a_{11}$ must be inserted in a cell immediately above the cell (including those in basement) containing $a_{11}$, the position of that cell fixes the value of $a_{11}$.

Now consider all the cells with $k$ and also the last cell in reading order among all those containing the entry $k-1$, then these cells are created by $U_{\lambda} \leftarrow a_{11} \ldots a_{1 c_{1}} a_{21}$. By Lemma 12, we know the corresponding row recording tableau and hence we know which two cells are the first two entries being inserted using the corresponding row word. Note that the first row consists of distinct entries and is inserted in ascending order using the row word, then by Lemma 15 in [HMR13], we know the cells are created in ascending reading order (one after another in reading order) and so they must be the cells immediately above those in $U_{\lambda}$, and hence the value inside each of those cells in the SSAF created by inserting the row word into $U_{\lambda}$ is exactly the value inside the cell just below it. Hence we know what the first two row entries of the corresponding SSAF of $a_{11} a_{12} \ldots a_{1 c_{1}} a_{21}$ are. Since we already know the first row entry, which is the lowest entry of the column corresponding to $a_{11} \ldots a_{1 c_{1}}$ is, we now know what the lowest entry of the second column (corresponding to $a_{11} \ldots a_{1 c_{1}} a_{21}$ and hence the same for $\left.a_{11} \ldots a_{1 c_{1}} a_{21} \ldots a_{2 c_{2}}\right)$ is.

We can repeat the same process until we get all the last entries of the $k$ columns and hence fix the shape of the SSAF corresponding to $w$. Since we read those entries just by considering $L$, this shows that $L$ fixes the shape of the corresponding SSAF of $w$ and result follows.

\section{References}

[HHL08] J. Haglund, M. Haiman, and N. Loehr. A combinatorial formula for nonsymmetric Macdonald polynomials. Amer. J. Math., 130(2):359-383, 2008.

[HLMvW11] J. Haglund, K. Luoto, S. Mason, and S. van Willigenburg. Refinements of the LittlewoodRichardson rule. Trans. Amer. Math. Soc., 363(3):1665-1686, 2011.

[HMR13] James Haglund, Sarah Mason, and Jeffrey Remmel. Properties of the nonsymmetric Robinson-Schensted-Knuth algorithm. J. Algebraic Combin., 38(2):285-327, 2013.

[Jos03] Anthony Joseph. A decomposition theorem for Demazure crystals. Journal of Algebra, 265(2):562 - 578, 2003.

[LS89] A. Lasku and M.-P. Shyuttsenberzhe. Noncommutative Schubert polynomials. Funktsional. Anal. i Prilozhen., 23(3):63-64, 1989.

[LS90] Alain Lascoux and Marcel-Paul Schützenberger. Keys \& standard bases. In Invariant theory and tableaux (Minneapolis, MN, 1988), volume 19 of IMA Vol. Math. Appl., pages 125-144. Springer, New York, 1990.

[Mac95] I. G. Macdonald. Symmetric functions and Hall polynomials. Oxford Mathematical Monographs. The Clarendon Press, Oxford University Press, New York, second edition, 1995. With contributions by A. Zelevinsky, Oxford Science Publications. 
[Mac96] I. G. Macdonald. Affine Hecke algebras and orthogonal polynomials. Astérisque, (237):Exp. No. 797, 4, 189-207, 1996. Séminaire Bourbaki, Vol. 1994/95.

[Mar99] Dan Marshall. Symmetric and nonsymmetric Macdonald polynomials. Ann. Comb., 3(24):385-415, 1999. On combinatorics and statistical mechanics.

[Mas08] S. Mason. A decomposition of Schur functions and an analogue of the RobinsonSchensted-Knuth algorithm. Sém. Lothar. Combin., 57:Art. B57e, 24, 2006/08.

[Mas09] S. Mason. An explicit construction of type A Demazure atoms. J. Algebraic Combin., 29(3):295-313, 2009.

[Wat14] M. Watanabe. Tensor product of Kraskiewicz and Pragacz's modules. ArXiv e-prints, October 2014. 